Role of Singular Layers in the Plasma Response to Resonant Magnetic Perturbations

F. L. Waelbroeck, I. Joseph, E. Nardon, M. Becoulet, R. Fitzpatrick

October 24, 2011

Nuclear Fusion 
This document was prepared as an account of work sponsored by an agency of the United States government. Neither the United States government nor Lawrence Livermore National Security, LLC, nor any of their employees makes any warranty, expressed or implied, or assumes any legal liability or responsibility for the accuracy, completeness, or usefulness of any information, apparatus, product, or process disclosed, or represents that its use would not infringe privately owned rights. Reference herein to any specific commercial product, process, or service by trade name, trademark, manufacturer, or otherwise does not necessarily constitute or imply its endorsement, recommendation, or favoring by the United States government or Lawrence Livermore National Security, LLC. The views and opinions of authors expressed herein do not necessarily state or reflect those of the United States government or Lawrence Livermore National Security, LLC, and shall not be used for advertising or product endorsement purposes. 


\title{
Role of Singular Layers in the Plasma Response to Resonant Magnetic Perturbations
}

\author{
F. L. Waelbroeck, ${ }^{1}$ I. Joseph,${ }^{2}$ E. Nardon, ${ }^{3}$ M. Bécoulet, ${ }^{3}$ R. \\ Fitzpatrick, ${ }^{1}$ \\ ${ }^{1}$ Institute for Fusion Studies, Univ. Texas at Austin, Austin TX 78712, USA \\ ${ }^{2}$ Lawrence Livermore National Laboratory, 7000 East Ave., Livermore, California \\ 94551, USA \\ ${ }^{3}$ Association Euratom/CEA, CEA Cadarache, F-13108, St. Paul-lez-Durance, France
}

\begin{abstract}
The response of an H-mode plasma to magnetic perturbations that are resonant in the edge is evaluated using a fluid model. With two exceptions, the plasma rotation suppresses the formation of magnetic islands, holding their widths to less than a tenth of those predicted by the vacuum approximation. The two exceptions are at the foot of the pedestal, where the plasma becomes more resistive, and at the surface where the perpendicular component of the electron velocity reverses. The perturbations exert a force on the plasma that is such as to brake the perpendicular component of the electron rotation. In the pedestal, the corresponding Maxwell stress drives the radial electric field in such a way as to accelerate ion rotation. Despite the suppression of the islands, the perturbations give rise to particle fluxes caused by magnetic flutter, with a negligible contribution from $\mathrm{E} \times \mathrm{B}$ convection. In the pedestal, the fluxes are such as to reduce the density.
\end{abstract}

PACS numbers: 52.30.Ex, 52.55.Tn, 52.35.Vd,52.40.Fd 
Role of Singular Layers in the Plasma Response to Resonant Magnetic Perturbations 2

\section{Introduction}

Edge Localized Modes (ELM) have the potential to cause an unacceptable erosion of the divertor in ITER [1] and future fusion reactors. In present-day experiments, these modes eject up to $20 \%$ of the energy stored in the edge pedestal. In ITER, by contrast, survival of the divertor will require limiting the amplitude of heat pulses to approximately $1 \%$ percent of the pedestal energy [1]. This has motivated the development of methods for mitigating ELMs.

An effective ELM mitigation method consists of applying resonant magnetic perturbations (RMP) to the edge [2]. Magnetic perturbations are said to be resonant when they induce a finite electromotive force on closed field-lines lying inside the plasma [3]. Resonant magnetic perturbations can be produced by currents flowing in coils external to the plasma, or by a saturated intrinsic mode such as the edge harmonic oscillation (EHO) [4, 5, 6]. In most experiments, such as those on COMPASS-D [7, JFT-2M [8], NSTX [9], JET [10,11] and DIII-D [12], the RMP cause the ELM to be excited at reduced pedestal energy, resulting in smaller and more frequent ELM. Remarkably, however, in low-collisionality experiments with in-vessel coils on DIII-D, the RMP can completely eliminate the ELM [13, 14, This has been observed, in particular, in discharges with shapes similar to those planned for ITER [15]. In these experiments the RMP acts by degrading the confinement in the edge, thereby reducing the width of the pedestal as well as its peak pressure gradient.

The effectiveness of the RMP method has motivated the design of an in-vessel coil system dedicated primarily to suppressing the ELM on ITER [1, 16, 17]. Three sets of coils are planned to be located behind the blanket shield module. Considerable uncertainties remain, however, concerning the required perturbation amplitude as well as the criteria for optimizing the mode spectrum. In addition to the differences in the observed effects of the RMP on different machines, there are important questions regarding the interpretation of the results, and in particular the role of magnetic chaos and of the response of the plasma to the perturbations. We next describe the two main paradigms for interpreting the effects of the RMP.

\subsection{The magnetic-chaos model}

The design of the RMP coils for ITER and the analysis of the RMP experiments have relied on a model that attributes the suppression to the presence of magnetic chaos 12, 18, $19,20,21$. According to this model, the RMP creates overlapping magnetic islands which degrade confinement, reducing the pedestal gradients and thereby stabilizing the ELM [14,22,23]. Almost all the experimental tests of this model, however, estimate the size of magnetic islands by superposing vacuum magnetic perturbations on the equilibrium. That is, they neglect the currents induced in the plasma by the RMP.

The magnetic chaos model has served as the main guide for the design of experiments. Several features of the observations have been interpreted as supporting this model, such as the enhanced transport and the splitting of the footprints on the 
divertor strike-plates. Simulations of transport in chaotic fields, however, show that the electron temperature pedestal is eroded and widened to an extent that is difficult to reconcile with experimental observations $24,25,26$. They also fail to account for the often-observed increase in the temperature gradient in the pedestal, and predict fluxes at the divertor strike plates that differ from observations by an order of magnitude. Lastly, the chaos model predicts that ELM suppression should occur when the edge safety factor $q_{95}$ falls in a window of width $\delta q_{95} \sim 1$, whereas in DIII-D, ELM suppression is only observed in a very narrow window $\delta q_{95} \sim 0.1$.

Explanations for these discrepancies have been proposed based on a number of effects, all of which are omitted from the present paper. First, Tokar and his collaborators have shown that heat flux limits can prevent the erosion of the temperature profile and in some cases even lead to profile steepening [18, 20]. The flux-limited transport model in these papers agrees with experiments even in the absence of screening. Tokar and his collaborators included the role of screening in a subsequent paper 27] in which they show that the deviations from neoclassical equilibrium induced by the RMP lead to radial ion flows that exceed significantly the ion fluxes caused by flow along the field lines. This allows their model to explain pump-out for the level of RMP screening specified by Ref. [28]. Note that Refs. [18,20,27] take into consideration particle sources and pinches. Lastly, Park et al. [29] have drawn attention to the role of trapped particles. They also found that a screening factor was necessary in order to successfully model the experimental results. Other simulations using MHD [30 and two-fluid models [31, 32 have shown that plasma rotation has the effect of reducing the width of pre-existing magnetic islands, thereby healing the magnetic chaos. These works have led to increased interest in models that account for the response of the plasma self-consistently.

\subsection{Onset of locked modes}

In the context of error fields and low- $n$ RMP ( $n$ is the toroidal mode number), the response of the plasma to a magnetic perturbation that is resonant in the core of the discharge is well understood. Experiments [33,34,35,36,37,38] show that plasma rotation greatly inhibits the growth of the RMP-driven magnetic islands. The RMP, however, exerts a braking torque on the plasma rotation [39,40]. For sufficiently large amplitude, a bifurcation occurs and the plasma near the resonant surface abruptly locks to the error field [33,41]. The resonant island subsequently grows rapidly to a size comparable to that of the corresponding vacuum island [42]. This bifurcation phenomenon has sometimes been referred to by the term "mode penetration," 33 but some confusion has been created by the alternative use of this term by some authors to describe the degree of spatial attenuation of the magnetic perturbation as it propagates inwards from the edge. In this paper, we will refer to the bifurcation as a locked-mode onset (LMO). The LMO exhibits several clearly identifiable signatures, such as (1) plasma spin-down, (2) a magnetic pulse, and (3) a prompt flattening of the electron temperature near the 
resonance. Furthermore, removal of the RMP leads to (4) a spin-up of the induced island that is easily observed on Mirnov coils.

The above phenomenology of the LMO is in qualitative agreement with MHD theory, which shows that the suppression of the island is associated with singular currents flowing near the resonant surface $43,44,45,46,41,47,48$. The bifurcation occurs when the viscous force on the resonant layer, which acts to maintain the rotation, can no longer balance the electromagnetic braking force created by the RMP.

Efforts to improve the quantitative predictions of the theory have led to the realization that the non-resonant components of the field also affect the plasma rotation by giving rise to a neoclassical toroidal viscosity (NTV), which exerts a braking force proportional to the deviation of the rotation velocity from a value determined by neoclassical effects $49,50,51,52$. Bécoulet et al. have examined the role of NTV forces in the context of ELM-suppression experiments [53]. Since the NTV and resonant forces are additive, however, in this paper we will restrict consideration to the resonant forces.

\subsection{Two-fluid theory of island suppression and of the locked-mode onset}

Before applying the error-field theory to the pedestal, one must face the fact that the conditions pertaining to the edge differ substantially from those that apply in the confinement region, where the dominant error-field $(n=1)$ resonances lie. First, the sharp gradients in the pedestal give rise to diamagnetic drifts that can exceed the electric $(\mathrm{E} \times \mathrm{B})$ drift. Second, the proximity of the separatrix results in high magnetic shear and strong shaping, particularly in the high-triangularity experiments with ITER-similar shapes (ISS) [15]. Third, the combination of steep pressure gradients and a higher toroidal mode number ( $n=3$ in DIII-D) leads to the likelihood of coupling between neighboring resonances. Lastly, one expects the distinctive nature of momentum transport in the edge to affect rotation braking by the RMP.

The effects on the plasma response of robust diamagnetic drifts and weak collisionality were first investigated in a general context, using singular layer theory, in Ref. [54]. This paper predicted that the locked mode threshold would be minimum for resonant surfaces such that the perpendicular component of the electron rotation vanishes, and that the RMP would modify rotation so as to brake the perpendicular electron rotation. This was subsequently confirmed by experiments on TEXTOR [55,56]. Note that in H-mode the perpendicular electron rotation is generally opposite to the ion rotation, so that electron braking entails ion acceleration. Around the same time as the TEXTOR experiments, Cole and Fitzpatrick [57] revisited the theory of Ref. [54] in an effort to explain the ALCATOR C-MOD observations of the scaling of the LMO threshold with density [58]. Their model generalized that of [54] by including the effects of finite ion temperature and particle diffusivity. Consistent with their focus on core-resonant modes, however, they neglected the diamagnetic frequencies compared to the plasma rotation frequency. Shortly thereafter Heyn et al. 28] used a kinetic model in cylindrical geometry to investigate a set of experiments with the dynamic 
Role of Singular Layers in the Plasma Response to Resonant Magnetic Perturbations 5

Table 1. Theoretical investigations of the plasma response. The papers marked with a dagger $\left({ }^{\dagger}\right)$ use locked modes as initial conditions. The paper marked with an asterisk $\left.{ }^{*}\right)$ use a periodic-slab geometry.

\begin{tabular}{|c|c|c|c|}
\hline & MHD & Two-fluid (Drift) Model & Kinetic model \\
\hline 1D (Layer) & $\begin{array}{c}\text { Fitzpatrick } \\
45,41\end{array}$ & $\begin{array}{c}\text { Fitzpatrick \& Hender, } 43 \\
\text { Waelbroeck, } 54 \\
\text { Cole \& Fitzpatrick, } \\
57\end{array}$ & \\
\hline 2D (cyl) & Kikuchi 48 & $\begin{array}{c}\text { Kikuchi, } 59 \text { Yu, } 60,63,64 \\
\text { Militello* } \\
\text { Nardon, } 62 \text { Bécoulet } 17\end{array}$ & Heyn 61 \\
\hline 3D (Tor.) & $\begin{array}{c}\text { Nardon, } 66] \\
\text { Izzo \& Joseph, }{ }^{\dagger} \\
\text { Liu }[67]\end{array}$ & $\begin{array}{c}\text { Strauss, }^{\dagger} 31 \\
\text { Reiser \& Chandra }^{\dagger} 32\end{array}$ & Park 29 \\
\hline
\end{tabular}

ergodic divertor (DED) on TEXTOR. Kikuchi et al. [59] also simulated the TEXTOR experiments using a reduced quasi-linear two-fluid model in cylindrical geometry and confirmed the predictions of strong screening and of braking of the electron rotation. Yu et al. [60] subsequently explained the asymmetry in the dependence of the TEXTOR LMO threshold on neutral beam power in terms of the modification of the temperature profile by the perturbation.

More recently, Heyn et al. [61] have applied their model to RMP experiments on DIII-D. Their results concluded that in DIII-D, to an even greater extent than in TEXTOR, islands are strongly suppressed, so that magnetic chaos does not extend across the pedestal during these experiments. Their calculation used a kinetic model with a Krook collision operator in cylindrical geometry. The cylindrical geometry makes it necessary to modify the current profile in order to mock-up the divergence in the safety-factor at the separatrix. Nardon et al. 62] used a two-fluid model, also in cylindrical geometry, to show that including electron compressibility and drifts in a fluid model leads to erosion of the density gradient in the pedestal for DIII-D-like parameters and profiles. Their results confirm the strong suppression (away from the electron rotation reversal surface) that was found in Ref. [61]. Table 1 provides a summary of numerical and analytic investigations on the plasma response to RMP. Note that the table as well as the preceding discussion omit all the research on the interaction of RMP with preexisting rotating magnetic islands such as those caused by tearing modes.

Applying the knowledge gained from error-field experiments and theory to interpret the results of RMP experiments, one expects that magnetic chaos will set in only after neighboring resonant surfaces in the pedestal undergo LMO. An exception to this rule is the narrow region at the foot of the pedestal, very near the separatrix, where the electron rotation is slow, the resistivity relatively high and the distance between resonant surfaces is less than the Larmor radius of the ions. In the rest of the pedestal, however, locked modes are deliberately avoided in ELM mitigation experiments since they would cause an undesirable transition to L-mode confinement [7]. In the present paper, we 
investigate explanations for the ELM-mitigation observations based on the predictions of a two-fluid model for the response of the plasma in the edge.

\subsection{Transport induced by suppressed RMP}

In order to evaluate the prospects of the coil system planned for ITER, it is clearly necessary to improve our understanding of the mechanisms whereby the RMP affect the stability of the edge. In this paper, we will examine the particle transport induced by screened RMP, as well as the effect of anomalous transport on the screening of the perturbation by the plasma. We use a two-fluid model for the singular layer that allows us to investigate the effects of anomalous transport phenomena, such as viscosity and particle diffusivity, that were neglected in Ref. [61]. Our model omits other transport effects, however, such as particle sources and pinches. Our use of a layer theory has the consequence that the magnetic shear in our model is free from the constraints imposed on cylindrical codes by the equilibrium model. Nevertheless, the results of our analysis are in broad agreement with those of cylindrical codes [17, 59, 61, 62].

The remainder of this paper is organized as follows. We begin in Sec. 2 by describing the model used in our analysis. We next examine in Sec. 3 the various quasilinear transport fluxes induced by the RMP. In Sec. 4, we describe the results of numerical calculations of the normalized torque induced by the resonant response to the perturbation. In particular, we examine the dependence of the normalized torque on some experimentally important parameters, and compare the numerical and analytic results. In Sec. 5, we apply our two-fluid model to the calculation of the reconnection quotients for a model of the edge profiles introduced in Ref. 62]. Lastly, we summarize and discuss the results in Sec. 6. In Appendix A, we describe some asymptotic analytic results, which are used in the paper to benchmark the numerical solutions.

\section{Formulation}

We describe the response of the plasma in the resonant layer by using the "fourfield" model of Hazeltine et al. 68, 69 We note that the the four-field model uses a finite Larmor radius (FLR) expansion for the ion dynamics $\left(k \rho_{i} \ll 1\right.$, where $\rho_{i}$ is the ion Larmor radius). In the low-collisionality regime of the DIII-D experiments, however, the resonant layers are narrower than the Larmor radius. Several studies of tearing and resistive kink growth rates have shown, however, that in the linear regime, the FLR assumption yields results in good agreement with calculations that account for the nonlocal nature of ion gyration (see 70,71 and references therein). Of greater consequence is the neglect of neoclassical effects and electron heat transport. Simulations using the XGC0 code 29,72 provide perspective on the role of these effects.

We adopt the following additional simplifications. First, we neglect the effects of field-line curvature. Second, we neglect electron inertia in Ohm's law. This assumption is violated for the resonant surfaces in the center of the pedestal, where the island 
suppression is the strongest. 61 Comparison of our results with those of Ref. 61] suggest that the effects of electron inertia are mild. Third, we assume that the resonant layer is sufficiently thin that the excitation of ion-acoustic waves is negligible, so that we may neglect the parallel compressibility of the ions. This assumption, which reduces the number of fields to three, is justified when $k_{\|} c_{s} \ll \omega_{*}$, where $k_{\|}=\mathbf{k} \cdot \mathbf{B}_{0} / B_{0}$ is the wavevector in the direction of the background magnetic field $\mathbf{B}_{0}, c_{s}=\left(T_{e} / m_{i}\right)^{1 / 2}$ is the ion-sound speed, and $\omega_{*}=k_{y} T_{e} / e B L_{n}$ is the drift frequency. Here, $k_{y}$ is the azimuthal wavevector, $T_{e}$ the constant electron temperature, $m_{i}$ the ion mass, and $L_{n}=n_{e} /\left|\nabla n_{e}\right|$ a scale-length describing the gradient of the electron density $n_{e}$. We note that Ref. 32] also used the four-field model but retained curvature, ion compressibility, and electron inertia. Their initial conditions, however, consisted of a plasma with fully reconnected islands, whereas the analysis presented here assumes that the RMP is applied to a plasma with initially good (simply nested) flux surfaces.

The state of the plasma is represented in terms of the normalized quantities $A, \varphi$ and $n_{e}$ as follows. The magnetic field is $\mathbf{B}=B_{0}(\hat{\mathbf{z}}-\hat{\mathbf{z}} \times \nabla A)$ where $\hat{\mathbf{z}}=\mathbf{B}_{0} / B_{0}$ is a unit vector in the direction of the magnetic field at the resonant surface of interest, and $A=\hat{\mathbf{z}} \cdot \mathbf{A}$ is the axial component of the magnetic potential, and the lengths are normalized to the minor radius $a$. The electric drift velocity is $\mathbf{v}_{E}=v_{A}(\hat{\mathbf{z}} \times \nabla \varphi)$, where $\varphi$ is the electrostatic potential and $v_{A}=B_{0} / \sqrt{\mu_{0} m_{i} n_{0} / Z}$ is the Alfvén velocity, $n_{0}$ is the (dimensional) electron density, and $\mathrm{Z}$ is the effective charge. The components along the background magnetic field of the normalized current and vorticity are $J=-\nabla^{2} A$ and $U=\nabla^{2} \varphi$, respectively. Lastly, the plasma density $n_{e}$ is normalized so that the diamagnetic electron drift velocity is $\mathbf{v}_{d e}=v_{A}\left(\hat{\mathbf{z}} \times \nabla n_{e}\right)$. Under the assumptions described above, the model describing the resonant layer reduces to the following equations describing the evolution of $\varphi, n_{e}$, and $A$ :

$$
\begin{aligned}
& \frac{\partial n_{e}}{\partial t}=-\mathbf{v}_{E} \cdot \nabla n_{e}+\rho_{s}^{2} \nabla_{\|} J+\mathcal{D} \nabla^{2} n_{e} \\
& \frac{\partial U}{\partial t}=-\mathbf{v}_{E} \cdot \nabla U-\nabla \cdot\left[\left(\mathbf{v}_{d i} \cdot \nabla\right) \nabla \varphi\right]+\nabla_{\|} J+\mu \nabla^{2}\left(U+\tau \nabla^{2} n_{e}\right) \\
& \frac{\partial A}{\partial t}=-\mathbf{v}_{E} \cdot \nabla A+\nabla_{\|} n_{e}-\eta J
\end{aligned}
$$

Here $\rho_{s}=c_{s} / a \omega_{c i}$ where $\omega_{c i}=e B_{0} / m_{i}$ is the ion cyclotron frequency, $\mathcal{D}$ and $\mu$, are the particle diffusivity and the viscosity coefficient, $S=\mu_{0} v_{A} / \eta$ is the Lundquist number, $\eta$ is the resistivity, $\mathbf{v}_{d i}=\tau \mathbf{v}_{d e} / Z$ is the ion diamagnetic velocity, and $\tau=T_{i} / T_{e}$. Note that if the transport is dominated by collisional processes, as assumed in Ref. [57], then $\mathcal{D}=\beta_{e} S$, where $\beta_{e}=2 \mu_{0} n T_{e} / B_{0}^{2}$ is the ratio of electron thermal and magnetic pressures. Here, we allow $\mathcal{D}$ to be independent of $S$ in order to model anomalous diffusive processes.

We consider a reference state consisting of an inhomogeneous plasma flowing in a sheared magnetic field. This reference state is described by

$$
\varphi_{0}=\omega_{E} x ; \quad n_{e 0}=-\omega_{*} x ; \quad A_{0}=x^{2} / 2\left(a / L_{s}\right),
$$

where $\omega_{E}$ is the $E \times B$ rotation frequency of the plasma in the laboratory frame and $L_{s}=R_{0} q / s_{s}$, where $s_{s}=d \log q /\left.d \log r\right|_{r=r_{s}}$ is the magnetic shear. In the singular 
layer, the azimuthal derivatives are small compared to the derivatives across the layer, $\partial / \partial y \ll \partial / \partial x$. It is convenient to rescale the variables by using the collisional layer width $\delta=\eta^{1 / 3}$ according to $X=x / \delta, T=t \delta, \rho=\rho_{s} / \delta$, and $\Xi=\xi / \delta^{2}$ where " $\Xi "$ symbolizes the new normalized fields. With these normalizations and using Poisson brackets defined by $[f, g]=\hat{\mathbf{z}} \cdot(\nabla f \times \nabla g)$, the equations take the form

$$
\begin{aligned}
& \frac{\partial n_{e}}{\partial T}=-\left[\varphi, n_{e}\right]+\rho^{2}[J, A]+D \nabla^{2} n_{e} \\
& \frac{\partial U}{\partial T}=-[\varphi, U]-\tau \nabla \cdot\left[n_{e}, \nabla \varphi\right]+[J, A]+P \nabla^{2}\left(U+\tau \nabla^{2} n_{e}\right) \\
& \frac{\partial A}{\partial T}=-\left[\varphi-n_{e}, A\right]-J
\end{aligned}
$$

where $P=\mu / \eta$ and $D=\mathcal{D} / \eta$, and $\nabla^{2}=\partial^{2} / \partial X^{2}$.

We seek solutions of the form

$$
\xi(X, y, t)=\bar{\xi}(X)+\Re\left[\tilde{\xi}(X) e^{i y-i \omega_{0} t}\right]
$$

where $\Re$ denotes the real part, $\xi=\left(n_{e}, \varphi, A\right)$ and where the perturbation amplitudes $\tilde{\xi}$ are assumed small compared to the $y$-averaged fields $\bar{\xi}: \tilde{\xi} \ll \bar{\xi}$. Here $\omega_{0}$ is the rotation frequency of the RMP in the lab frame. The primary goal of our analysis is to evaluate the change of the background fields

$$
\bar{\xi}=\xi_{0}+\delta \xi,
$$

where $\xi_{0}$ describes the background field before the application of the RMP. This goal is achieved in two steps. In the first step, described in Sec. 2.1, we evaluate the linear response $\tilde{\xi}$ of the plasma to the applied RMP. In the second step, described in Sec. 3 . we use the linear response to evaluate the quasilinear modifications to the background profiles.

\subsection{Linear theory of the singular-layer response}

To lowest order in the RMP amplitude, the perturbations are described by the linearized equations of motion.

$$
\begin{array}{ll}
Q \tilde{n}_{e}-Q_{*} \tilde{\varphi}-i X \rho^{2} \tilde{A}^{\prime \prime} & =D \tilde{n}_{e}^{\prime \prime} ; \\
Q_{i} \tilde{\varphi}^{\prime \prime}-i X \tilde{A}^{\prime \prime} & =P\left(\tilde{\varphi}+\tau \tilde{n}_{e}\right)^{\prime \prime \prime \prime} ; \\
Q_{e} \tilde{A}-i X\left(\tilde{\varphi}-\tilde{n}_{e}\right) & =\tilde{A}^{\prime \prime} .
\end{array}
$$

where the primes represent radial derivatives, $\xi^{\prime}=d \xi / d X, Q=-i\left(\omega_{0}-\omega_{E}\right) / \gamma_{r}$, $Q_{*}=-i \omega_{*} / \gamma_{r}$, where $\gamma_{r}=\delta^{1 / 3}$ is the reconnection rate for a mode that is marginally stable to an ideal mode, such as the internal kink. Lastly, $Q_{i}=Q+\tau Q_{*}$, and $Q_{e}=Q-Q_{*}$. At large distances from the resonant layer, $X \gg 1$, the solutions of the layer Eqs. (8)-(10) must be matched to the solution outside the layer which takes the form

$$
\begin{aligned}
& \tilde{A} \sim \tilde{A}_{0}+\tilde{A}_{1}|X|+O\left(X^{2}\right) \\
& \tilde{\varphi} \sim-i Q\left(\tilde{A}_{0} X^{-1}+\tilde{A}_{1} \operatorname{sign}(X)\right) ; \\
& \tilde{n}_{e} \sim\left(Q_{*} / Q\right) \tilde{\varphi} .
\end{aligned}
$$


Role of Singular Layers in the Plasma Response to Resonant Magnetic Perturbations 9

The result of the linear solution of the layer equations is contained in the quantity $\hat{\Delta}$ that represents jump in the ratio of the coefficients of the small and large terms in the asymptotic expansion:

$$
\hat{\Delta}(Q)=2 \tilde{A}_{1} / \tilde{A}_{0}
$$

In the following section, we will extend previous calculations of $\hat{\Delta}(Q)$ to the present model.

\subsection{Ballooning transformation}

The equations (8)-(10) represent an 8th order system of ordinary differential equations. Fortunately, Fourier transforming these equations reduces the order of the system to two. Note that the Fourier transform variable $k$ is proportional to the ballooning angle $[73$. That is, $k$ measures the distance along the field line. When interpreting the wavefunctions in ballooning coordinates it is useful to recall that, due to magnetic shear, quantities that vary slowly along the field lines develop strong radial gradients. As a result, dissipation becomes increasingly important at large $k$.

The transformed equations are

$$
\begin{aligned}
Q \hat{n}_{e}-Q_{*} \hat{\varphi}-\rho^{2} \frac{d \hat{J}}{d k} & =-D k^{2} \hat{n}_{e} \\
Q_{i} k^{2} \hat{\varphi}+\frac{d \hat{J}}{d k} & =-P k^{4}\left(\hat{\varphi}+\tau \hat{n}_{e}\right) \\
Q_{e} \hat{A}+\frac{d}{d k}\left(\hat{\varphi}-\hat{n}_{e}\right) & =-\hat{J} .
\end{aligned}
$$

where $\hat{J}=k^{2} \hat{A}$ is the current. Eliminating the current between the electron continuity and the vorticity equation yields the ion continuity equation,

$$
Q \hat{n}_{e}-Q_{*} \hat{\varphi}+Q_{i} k^{2} \rho^{2} \hat{\varphi}=-D k^{2} \hat{n}_{e}-P k^{4} \rho^{2}\left(\hat{\varphi}+\tau \hat{n}_{e}\right),
$$

which can be used to express $\hat{n}_{e}$ in terms of $\hat{\varphi}$. In order to reduce the system of equations (15)-17) to a single second order equation, it is convenient to introduce the electron stream function $\hat{\Upsilon}=\hat{\varphi}-\hat{n}_{e}$. Equation 18 makes it possible to express $\hat{\varphi}$ and $\hat{n}_{e}$ in terms of $\hat{\Upsilon}$ :

$$
\begin{aligned}
& \hat{n}_{e}=\frac{Q_{*}-Q_{i} \rho^{2} k^{2}-P \rho^{2} k^{4}}{Q_{e}+\left(D+Q_{i} \rho^{2}\right) k^{2}+(1+\tau) \rho^{2} P k^{4}} \hat{\Upsilon} ; \\
& \hat{\varphi}=\frac{Q+D k^{2}+\tau P \rho^{2} k^{4}}{Q_{e}+\left(D+Q_{i} \rho^{2}\right) k^{2}+(1+\tau) \rho^{2} P k^{4}} \hat{\Upsilon} .
\end{aligned}
$$

From Ohm's law (17) we further find that

$$
\hat{A}=-\frac{1}{k^{2}+Q_{e}} \frac{d \hat{\Upsilon}}{d k} .
$$

Substituting these result into either the continuity or vorticity equation, we obtain

$$
\frac{d}{d k}\left(\frac{k^{2}}{k^{2}+Q_{e}} \frac{d \hat{\Upsilon}}{d k}\right)=\left(\frac{Q Q_{i}+Q_{i}(D+P) k^{2}+P D k^{4}}{Q_{e}+\left(D+Q_{i} \rho^{2}\right) k^{2}+(1+\tau) \rho^{2} P k^{4}}\right) k^{2} \hat{\Upsilon} .
$$


This is a second-order differential equation governing the plasma response in the singular layer. Aside from minor differences in notation, it is essentially equivalent to that used in Ref. [57].

Pegoraro and Schep [74] have shown that in some regimes it is more convenient to solve for the current instead of the electron streamfunction $\hat{\Upsilon}$. Using Eq. (21), it is easy to obtain the equation for the current in the layer,

$$
\frac{d}{d k}\left(\frac{Q_{e}+\left(D+Q_{i} \rho^{2}\right) k^{2}+(1+\tau) \rho^{2} P k^{4}}{\left(Q Q_{i}+Q_{i}(D+P) k^{2}+P D k^{4}\right) k^{2}} \frac{d \hat{J}}{d k}\right)-\left(\frac{k^{2}+Q_{e}}{k^{2}}\right) \hat{J}=0 .
$$

In the inviscid limit, this form of the layer equation admits analytic solutions in both the collisional and semi-collisional regimes $[74]$. Solutions of the layer equations in asymptotic regimes are described in Appendix A.

\subsection{Asymptotic matching in ballooning space}

The matching conditions on the current require calculating the higher order terms in the asymptotic expansion of the solution, since the lowest-order terms vanish. For large $X$, the mode equations reduce to $Q \tilde{n}_{e}=Q_{*} \tilde{\varphi}, \tilde{A}=i(X / Q) \tilde{\varphi}$, and

$$
\frac{d}{d X}\left[\left(X^{2}+Q Q_{i}\right) \frac{d \tilde{\varphi}}{d X}\right]=0
$$

The first two terms of the series solution are

$$
\tilde{\varphi}(X)=-i Q \tilde{A}_{0} X^{-1}\left(1-\frac{Q Q_{i}}{3} X^{-2}+O\left(X^{-4}\right)\right)
$$

It follows that $\tilde{\varphi} \sim-i Q \tilde{A} / X$, where

$$
\tilde{A} \sim \tilde{A}_{0}\left(1-\frac{Q Q_{i}}{3} X^{-2}+O\left(X^{-4}\right)\right)+\tilde{A}_{1}\left(|X|+O\left(X^{-1}\right)\right) ;
$$

We obtain the small- $k$ asymptotic behavior of the layer response in ballooning space by taking the Fourier transform of the asymptotic expansion (24), after introducing the regularizing factor $\exp (-\epsilon|X|)$, and subsequently taking the limit $\epsilon \rightarrow 0$. There follows

$$
\begin{aligned}
& \hat{\Upsilon} \sim-Q_{e}\left(\frac{\tilde{A}_{0}}{2} \operatorname{sign}(k)+\frac{\tilde{A}_{1}}{\pi} k^{-1}+O(k)\right) ; \\
& \hat{J} \sim \tilde{A}_{0} \frac{Q Q_{i}}{6}|k|^{3}-\frac{\tilde{A}_{1}}{\pi} .
\end{aligned}
$$

The above matching conditions determine the matching parameter $\hat{\Delta}(Q)$, which itself determines the screening of the perturbation and the braking torque on the plasma.

\section{Quasilinear fluxes}

To evaluate the effect of the resonant magnetic perturbations on the background fields, we average the steady-state $(\partial / \partial t=0)$ layer equations along the azimuthal direction $y$. We use the identity

$$
\langle[f, g]\rangle=\frac{d}{d X}\left\langle f \partial_{y} g\right\rangle,
$$


Role of Singular Layers in the Plasma Response to Resonant Magnetic Perturbations11

where $\partial_{y}=\partial / \partial y$ and

$$
\langle f\rangle=\oint \frac{d y}{2 \pi} f(X, y)
$$

Taking the azimuthal averages and integrating once across the layer, we find

$$
\begin{array}{ll}
\left\langle\varphi \partial_{y} n_{e}\right\rangle-\rho^{2}\left\langle J \partial_{y} A\right\rangle & =D\left(\bar{n}_{e}^{\prime}+Q_{*}\right) ; \\
\left\langle\varphi \partial_{y} U\right\rangle-\left\langle J \partial_{y} A\right\rangle+\tau \frac{d}{d X}\left\langle n_{e} \partial_{y} \partial_{X} \varphi\right\rangle & =P \bar{V}_{i}^{\prime \prime} ; \\
\left\langle\left(\varphi-n_{e}\right) \partial_{y} A\right\rangle & =\bar{A}^{\prime}-X ;
\end{array}
$$

where $\bar{V}_{i}=d\left(\varphi+\tau n_{e}\right) / d X$ is the background ion velocity (the sum of the electric and diamagnetic drifts). Substituting the form (7) of the solution and carrying out the average along $y$, there follows

$$
\begin{array}{ll}
\frac{1}{2} \Im\left[\tilde{\varphi} \tilde{n}_{e}^{*}-\rho^{2} \tilde{J} \tilde{A}^{*}\right] & =D\left(\bar{n}_{e}^{\prime}+Q_{*}\right) ; \\
\frac{1}{2} \Im\left[\tilde{\varphi} \tilde{U}^{*}+\tau\left(\tilde{n}_{e} \tilde{\varphi}^{\prime *}\right)^{\prime}-\tilde{J} \tilde{A}^{*}\right] & =P \bar{V}_{i}^{\prime \prime} ; \\
\frac{1}{2} \Im\left[\left(\tilde{\varphi}-\tilde{n}_{e}\right) \tilde{A}^{*}\right] & =\bar{A}^{\prime}-X ;
\end{array}
$$

where $\xi^{*}$ is the complex conjugate of $\xi$ and $\Im$ denotes the imaginary part. The above equations may be simplified by noting that

$$
\Im\left[\tilde{\xi}^{*} \tilde{\xi}^{\prime \prime}\right]=\Im\left[\tilde{\xi}^{*} \tilde{\xi}^{\prime}\right]^{\prime} \text {. }
$$

We may use this identity to integrate the vorticity equation, Eq. (31), across the layer. This yields the well-known force-balance equation,

$$
\frac{1}{2} \Im\left[\tilde{A}^{\prime} \tilde{A}^{*}\right]=P\left[\left[\bar{V}_{\perp i}^{\prime}\right]\right] ;
$$

where $[[\cdot]]$ represents the jump across the layer and we have used the asymptotic property $\tilde{\varphi} \sim \tilde{n}_{e}=O\left(X^{-2}\right)$ to drop the term $\Im\left[\left(\tilde{\varphi}+\tau \tilde{n}_{e}\right) \tilde{\varphi}^{\prime *}\right]$. Equation 33 expresses the fact that in steady state, the $\mathbf{J} \times \mathbf{B}$ forces acting in the resonant layer must be balanced by a viscous force related to the jump in the gradient of the background velocity across the layer.

We next turn our attention to the continuity equation. The two terms on the left-hand side of Eq. (30) correspond to advective particle fluxes caused by, respectively

(i) eddies, created by the interaction of the RMP with the rotating plasma, that carry particles across the magnetic field and

(ii) electron currents along the perturbed magnetic field (magnetic flutter) that neutralize the ion polarization currents.

The possible role of the driven eddies in accounting for the effect of RMPs on the density was first noted by Nardon et al. [66], who observed that in an MHD model the scaling of this term with the resistivity resulted in it being too small to account for observations, given the small resistivity in experiments. Taking into account electron compressibility, however, has the effect of introducing the ion-sound Larmor radius $\rho_{s}$, thereby placing a lower bound on the decrease of the singular layer thickness with $\eta$. Because of its neglect of $\rho_{s}$, MHD may underestimate the effect of the eddies on the density. The 
second term in Eq. 30 is a two-fluid effect, as indicated by the $\rho^{2}$ coefficient. It is identical in form to the $\mathbf{J} \times \mathbf{B}$ force, so that we conclude that the same effect that gives rise to the resonant force in MHD also gives rise to a density jump across the layer.

In order to evaluate the effect of the quasilinear fluxes on the density, we integrate Eq. (30) radially across the layer and express the resulting pumpout as

$$
\left[\left[\bar{n}_{e}\right]\right]=\mathcal{V}_{\text {flutt }}+\mathcal{V}_{\text {conv }}
$$

where

$$
\mathcal{V}_{\text {flutt }}=\rho^{2} \Im\left[\left[\tilde{A}^{\prime} \tilde{A}^{*}\right]\right] / 2
$$

is the outflow velocity due to the magnetic flutter transport and

$$
\mathcal{V}_{\text {conv }}=\frac{1}{2} \int_{-\infty}^{\infty} d x \Im\left[\tilde{\varphi} \tilde{n}_{e}^{*}\right]
$$

is the outflow velocity due to the eddies. In terms of the wavefunctions in ballooning space, the latter term takes the form

$$
\mathcal{V}_{\text {conv }}=-\frac{1}{4 \pi} \int_{-\infty}^{\infty} d k \mathcal{W}(k)|\hat{\Upsilon}|^{2}
$$

where the weight function $\mathcal{W}$ is

$$
\mathcal{W}(k)=\Im\left[\frac{Q_{*}-Q_{i} \rho^{2} k^{2}-P \rho^{2} k^{4}}{Q_{e}+\left(D+Q_{i} \rho^{2}\right) k^{2}+(1+\tau) \rho^{2} P k^{4}}\right] .
$$

The integrand in (37) is regular at the origin and integrable at large $k$, as can be seen from the following alternative expression of $\mathcal{W}$ :

$$
\mathcal{W}(k)=-i k^{2} \frac{\left(Q_{*}-Q_{i} \rho^{2} k^{2}\right) D+P Q_{i} \rho^{2} k^{2}\left(1-\tau \rho^{2} k^{2}\right)}{\left(D+(1+\tau) P \rho^{2} k^{2}\right)^{2} k^{4}-\left(Q_{e}+Q_{i} \rho^{2} k^{2}\right)^{2}}
$$

The above form of the weight function shows that the perpendicular (eddy-driven) pinch vanishes if $D=0$ and either $P=0$ or $\rho=0$.

Our numerical calculations show that the eddy-driven flux never exceeds a few percent of the flutter flux for the parameters of interest in experiments, so we will henceforth neglect it. We note that Beyer et al. 75] have shown that in toroidal geometry the eddy-driven flux dominates over the parallel transport. The cause of the apparent conflict between the conclusions of that paper and the conclusions reached in the present paper is uncertain. One possibility is that curvature enhances the convective flux, in which case the present paper will underestimate the pump-out in toroidal experiments. Another possibility is that the electrostatic approximation used in [75] causes it to underestimate the flutter flux. Answering the question of the relative importance of the current-mediated flutter flux, which is the dominant transport mechanism in our model, and the eddy-mediated flux, which is the dominant transport mechanism in [75], requires a toroidal two-fluid calculation and falls outside the scope of the present paper.

Due to the singularity of $A(k)$ and unlike the eddy-driven flux, the pumpout caused by the magnetic flutter cannot be calculated by a Fourier space integral, but its value is easily determined by integrating across the layer. In fact, comparison of Eqs. (35) and 
(33) shows that the flutter-induced pumpout is proportional to the braking caused by the RMP:

$$
\left[\left[\bar{n}_{e}\right]\right]=\rho^{2} P\left[\left[\bar{V}_{\perp i}^{\prime}\right]\right] / D .
$$

This reflects the fact that the flutter pumpout results from the same radial currents that exert the resonant braking force. It can thus be thought of as a resonant radial pinch. Restoring the dimensions, the above identity takes the form

$$
\frac{\left[\left[\bar{n}_{e}\right]\right]}{n_{0}}=\frac{\mu}{D} \frac{\left[\left[\bar{V}_{\perp i}^{\prime}\right]\right]}{\omega_{c i}}
$$

For a given equilibrium and RMP spectrum, the right hand side of the above expression is proportional to the acceleration of the plasma in the edge. Eq. (40), however, must be corrected for the presence of neoclassical forces. We consider this next.

Following an argument due to Fitzpatrick 45 and recently applied to the particle transport problem by $\mathrm{Yu}$ and Günter [64], the above formula must be amended to account for the presence of neoclassical forces that strongly damp deviations of the poloidal velocity from its unforced value. As a result of these forces, the change in velocity caused by the RMP is almost entirely in the toroidal direction, $\delta \mathbf{V}=\delta \bar{V}_{\zeta} \mathbf{e}_{\zeta}$, where $\delta \bar{V}_{\zeta}=\delta \bar{V}_{\perp} B_{\zeta} / B_{\theta}$. The corresponding viscous force must balance the toroidal component of the perpendicular electromagnetic force, $T_{\zeta}=T_{E M} B_{\theta} / B_{\zeta}$. Force balance is thus achieved when

$$
\frac{\left[\left[\bar{n}_{e}\right]\right]}{n_{0}}=\frac{\mu}{D} \frac{\left[\left[\bar{V}_{\perp i}^{\prime}\right]\right]}{\omega_{c i}}\left(\frac{B_{\zeta}}{B_{\theta}}\right)^{2} .
$$

A more perspicuous form of the force-balance condition follows from the estimate $\left[\left[\bar{V}_{\perp i}^{\prime}\right]\right]=-\delta \bar{V}_{\perp i} / L_{p} N$, where $\delta \bar{V}_{\perp i}, L_{p}$, and $N$ are respectively the change of the perpendicular velocity, the pressure gradient scale-length (serving as an estimate of the pedestal width), and the number of resonant surfaces in the pedestal (for an $n=3 \mathrm{RMP}$ in DIII-D, $N \simeq 6$ ). The total pumpout caused by all the resonances in the pedestal is thus

$$
\frac{\left[\left[\bar{n}_{e}\right]\right]}{n_{0}}=-\frac{\mu}{D} \frac{\delta \bar{V}_{\perp i}}{V_{* i}}\left(\frac{\rho_{\theta}}{L_{p}}\right)^{2} .
$$

where $\rho_{\theta}=\left(B_{\zeta} / B_{\theta}\right) \rho_{s}$ is the poloidal gyroradius. We next discuss the implications of the above estimate, which is one of the most important results of the present paper.

The first implication of the above result, Eq. (42), is that it predicts that when the perpendicular electron velocity is in the electron diamagnetic direction, the pedestal density will decrease as a result of the application of an RMP, as observed in experiments, even in the absence of magnetic chaos. Ivanov et al. [76] reached similar conclusions with regards to changes in plasma rotation, pointing out that the explanation for the acceleration of the edge in terms of losses along open field lines in a chaotic magnetic field is fundamentally indistinguishable from the acceleration caused by isolated resonances: both are caused by the radial current induced by the RMP, and the nature of the mechanism does not change when neighboring islands overlap. 
Another interesting feature of our result, Eq. (42), is that although it predicts density pumpout in the edge, where the perpendicular rotation of electrons and ions is in opposite directions, it also predicts inwards particle fluxes at resonances in the core where the electric drift dominates, so that electrons and ions rotate in the same direction. Upon application of an RMP, in other words, the core plasma inhales. The magnitude of the pump-in may be determined from Eq. (41) in the same way as for the edge, except that the scale length for the change in density is now given by the much larger distance between the resonant surface and the edge, so that the magnitude of the effect is smaller. A similar effect was predicted for magnetic islands locked to the wall in Ref. [77], and was later demonstrated through numerical simulations in Ref. 64]. The effect may be understood as a consequence of the frozen-in law for electrons: since the electrons are unable to cross the separatrix once a magnetic island exceeds the tearing layer width, the existence of an electric field within the separatrix, whether from neutral beams or other causes, implies the presence of a diamagnetic drift that cancels the electric drift [3]. The formation of the pressure-gradient that creates the diamagnetic drift gives rise to a pumpout or a pump-in, according to the sign of the electric field.

The most significant implication of the above result relates to the scaling of the pumpout with the pedestal magnetization parameter $\rho_{\theta} / L_{n}$. Whether the width of the pedestal is determined by neutral penetration [78] or stability considerations [79], it is generally believed that its magnetization parameter will be smaller in ITER than in present-day machines. This raises the possibility that ELM suppression would be ineffective if it depends on the density pumpout. It should be noted, however, that pumpout occurs in nearly all RMP experiments, while ELM suppression is much more difficult to achieve. In DIII-D, in particular, the window in the edge safety factor $q_{95}$ for the suppression of the ELM is very narrow. Thus, while it is clear that the pumpout is insufficient to obtain suppression, it is unclear whether it is necessary.

We next describe the results of numerical solutions of the layer equation and compare these to the analytic results where appropriate.

\section{Numerical Studies}

In this section we present numerical solutions of the resonant layer equation for parameters relevant to the DIII-D experiment. In particular, we examine the dependence of the screening on particle diffusivity, on the viscosity, and on the collisionality.

\subsection{Effect of particle diffusivity}

In the early work on the plasma response to RMP [43, 54], the island suppression factor was found to exhibit three resonances when the perturbation frequency matched the Doppler frequencies of the ions, the electrons, and the guiding centers (which is

identical to the $E \times B$ frequency in a slab model). Subsequent numerical $[59,60,63,65]$ 
Role of Singular Layers in the Plasma Response to Resonant Magnetic Perturbations15

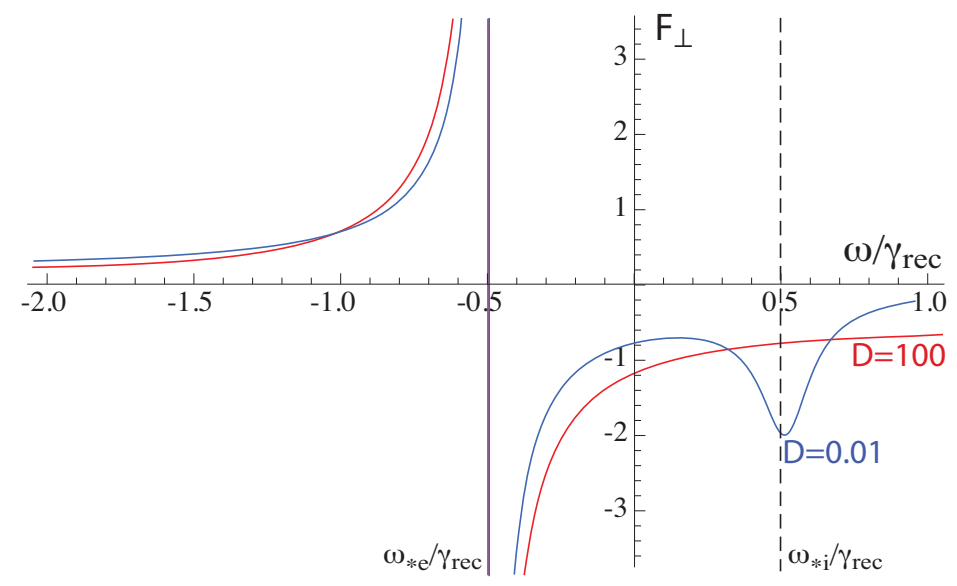

Figure 1. Effect of the particle diffusivity on the ion resonance in the electromagnetic force acting on the resonant layer.

and experimental [56, 55] investigations have found only a single resonance at the electron Doppler frequency. We have found that particle diffusivity is responsible for the disappearance of the other resonances. Fig. 1 shows the normalized force $F_{\perp}=\Im[1 / \hat{\Delta}(Q)]$ as a function of the normalized frequency $\omega / \gamma_{r}=i Q$. the ion resonance is extremely sensitive to particle diffusivity, so that even very small diffusivity will regularize the response.

\subsection{Effect of viscosity}

The various investigations of the plasma response to RMP have used values of the viscosity that vary from zero to two orders of magnitude above the turbulent viscosity, making comparisons difficult. For example, although it includes a model for Landau damping, the MARS-F code neglects viscosity entirely [67]. Likewise, the code used by Heyn et al. [61] does not account for diffusive processes. The neoclassical edge transport code $\mathrm{XGC0}$, by contrast, does model the turbulent transport as a radial random walk [29]. Lastly, most of the MHD and two-fluid calculations, including the present work, use spatially uniform estimates of the viscosity based on anomalous transport. $\mathrm{Yu}$ et al., however, have used artificially enhanced values of the viscosity in some of their work to model the effect of poloidal rotation damping on the balance between viscous and electromagnetic forces [63, 64]. While this approach models faithfully the zonal $(m=0)$ components of the flow, it overestimates by two orders of magnitude the viscosity experienced by the resonant $(m=n q)$ components.

It is thus of interest to examine the dependence of the plasma response on the viscosity to gain insight into the effects of the wide disparities in viscosity found in the literature. Fig. 2 shows the effect of the viscosity on the normalized force $F_{\perp}=\Im[1 / \hat{\Delta}(Q)]$ in the collisional limit defined by $\rho=0$. The continuous lines show the results obtained by integrating Eq. 22 numerically for two different values of the Prandtl number, $P=200$ and $P=0.2$. The remaining lines show the analytic results 
Role of Singular Layers in the Plasma Response to Resonant Magnetic Perturbations16

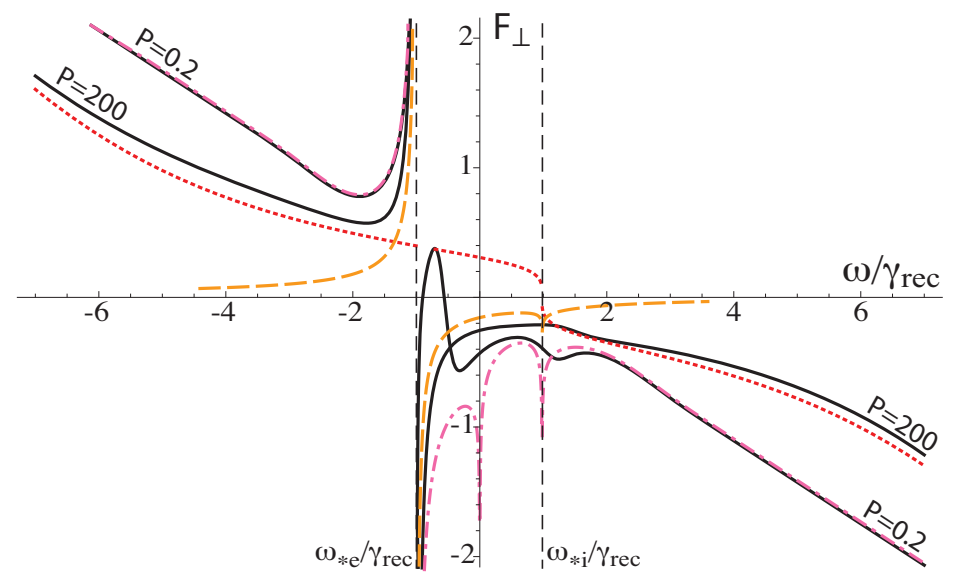

Figure 2. (color online) Effect of the viscosity and particle diffusivity on the electromagnetic force $F_{\perp}$ acting on plasma near the resonant surface, in the collisional limit $(\rho=0)$. The parameters are $\omega_{*} / \gamma_{r}=1, \tau=0$, and $D=P / 2$, where $P$ is indicated in the Figure.

for the inviscid regime, given in Eq. A.6) (dash-dotted line); the visco-resistive limit in Eq. A.9) (dashed line); and the ideal limit Eq. (A.2) (dotted line). Note that the visco-resistive limit only applies for very small rotation frequencies, $Q_{e} \ll\left(P / Q_{i}\right)^{-1 / 2}$, in agreement with the analytic estimate for the regime boundary. The Figure shows that in the collisional regime, for the frequencies of interest in experiments $(Q \sim 1)$, changing the viscosity by a factor of a thousand only changes the force by a factor of order unity.

We next consider the effect of viscosity in the low-collisionality regime relevant to DIII-D. Fig.3 shows the normalized force for a Larmor radius equal to 5 times the resistive layer width, $\rho=5$. The continuous lines show the results obtained by integrating Eq. 22 numerically for $P=200$ and $P=0.2$, with $D=P / 2$. The other lines show the analytic result in the ideal limit described by Eq. (A.2) (dotted line), the inviscid semi-collisional regime given in Eq. (A.14) (dashed line), and the diffusive semi-collisional regime described in Eq. (A.16) (dash-dotted). We see that in the lowcollisionality regime, the normalized force is much more sensitive to the anomalous transport parameters than in the collisional regime. This can also be seen by dividing the matching parameters given the analytic results in Eqs. (A.14) and A.16,

$$
\frac{\hat{\Delta}(D=0)}{\hat{\Delta}(D \gg 1)} \simeq 1.5\left(\frac{Q^{2}(1+\tau)}{D \rho^{2}}\right)^{1 / 4} .
$$

In the semi-collisional regime, $\rho \gg 1$ so that the above ratio is small. The effect on the screening factor, given by $|\hat{\Delta}|^{-1}$ in the constant- $A$ regime, is similar.

A noteworthy feature of Fig. 3 is the oscillations in the force for large values of the frequency. These are caused by resonances with kinetic Alfvén waves, and were studied previously by Drake et al. [80] in the inviscid regime $(P=0)$. Note that similar oscillations occur for $\omega>0$. These were left out of the range of Fig. 3 to improve the readability of the graph for small $\omega$. The large- $\omega$ oscillations may be of importance 
Role of Singular Layers in the Plasma Response to Resonant Magnetic Perturbations17

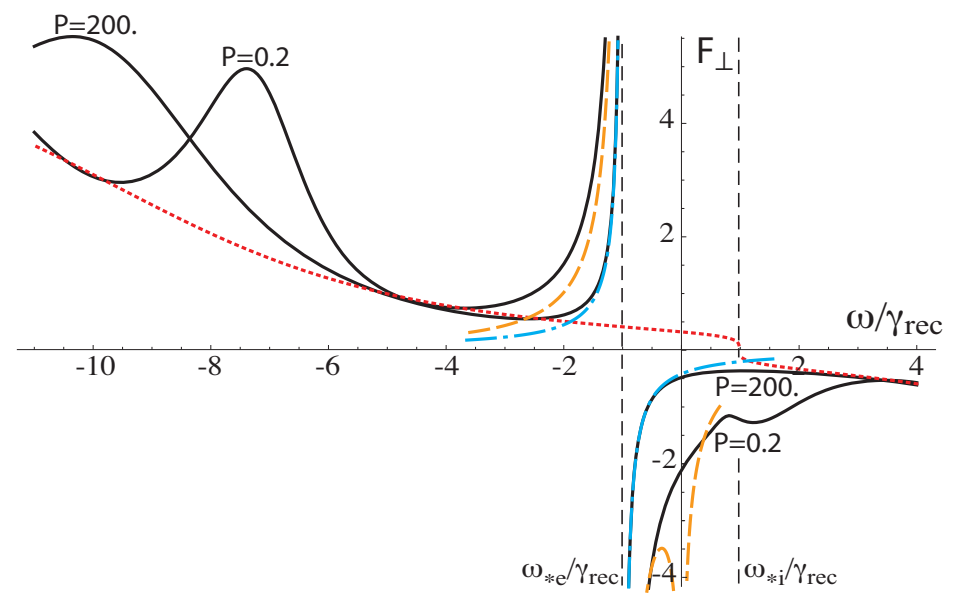

Figure 3. (color online) Effect of the viscosity and particle diffusivity on the electromagnetic force acting on the resonant layer in the semi-collisional regime for $\rho=6$, a value representative of the DIII-D pedestal. The parameters are $\omega_{*} / \gamma_{r}=1$, $\tau=1$, and $D=P / 2$, where $P$ is indicated in the Figure

in the core of rapidly rotating plasmas, or near shear-reversal surfaces. In the edge of fusion experiments, however, the value of $i Q=\omega / \gamma_{\text {rec }}$ rarely exceeds unity.

\subsection{Collisionality and the effect of electron compressibility}

Another important parameter influencing the plasma response is the electron compressibility, measured by $\rho$, which represents the ratio of the Larmor radius to the resistive layer width. We have seen in the previous Subsection that the electron compressibility can strongly influence the sensitivity of the results to the anomalous transport coefficients. Fig. 4 compares the dependence of the force $F_{\perp}$ on the normalized frequency $\omega / \gamma_{\text {rec }}$. The two solid lines show the results obtained by integrating Eq. 22 numerically for $\rho=0.0$ and $\rho=5.0$. The other lines show the analytic result in the ideal limit described by Eq. A.2 (dotted line), the visco-resistive regime given in Eq. (A.9) (short-dashed line), and the diffusive semi-collisional regime described in Eq. A.16) (long dashed line). The Figure shows that for small values of $\left(\omega-\omega_{* e}\right) / \gamma_{\mathrm{rec}}$, where the effects of the RMP are strongest, neglecting two-fluid effects can lead to a substantial underestimation of the amplitude of the resonant force $F_{\perp}$. For larger rotation frequencies, however, the effect is negligible.

Lastly, we note that the effect of neglecting $\rho$ compensates the effect of neglecting $P$ and $D$ that was described in the previous subsection. As a result, the predictions of inviscid resistive-MHD codes do not differ greatly from those of two-fluid models with anomalous transport coefficients.

\subsection{Effect of Low-shear}

We conclude this Section with a brief note on the effect of magnetic shear. An interesting feature of some equilibrium reconstructions is the presence of a narrow region towards 
Role of Singular Layers in the Plasma Response to Resonant Magnetic Perturbations18

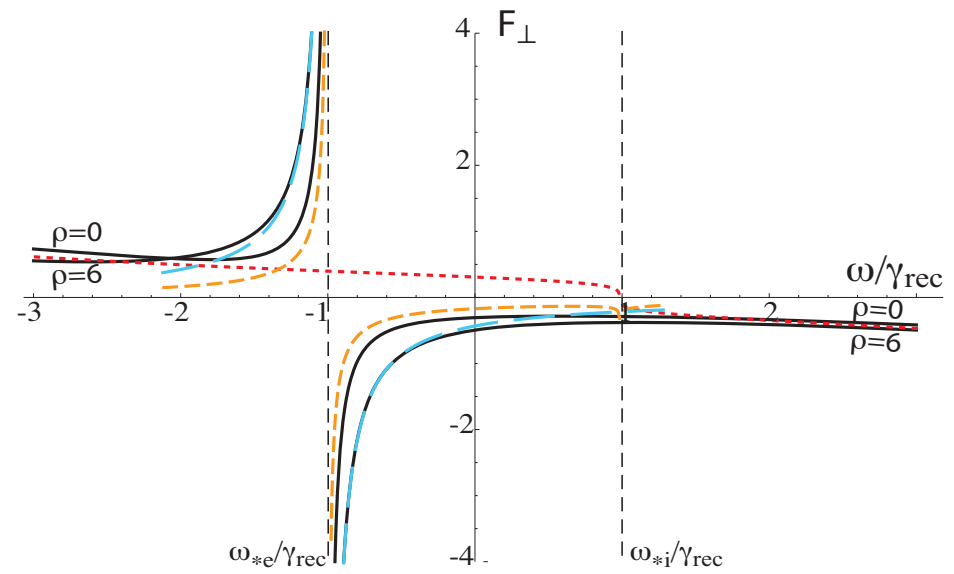

Figure 4. (color online) (color online) Comparison of the electromagnetic force acting on the resonant layer in the collisional limit $(\rho=0)$ and for a semi-collisional case with $\rho=6$, a value representative of the DIII-D pedestal. The other parameters are $\tau=1$, $P=2 D=200$ and $Q_{*}=1$

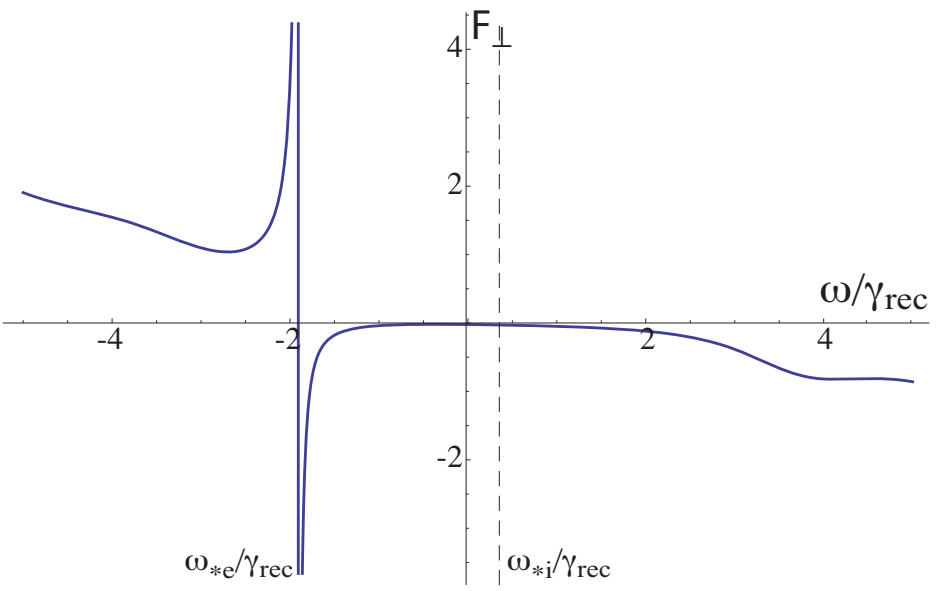

Figure 5. (color online) Normalized electromagnetic torque for a resonance surface with weak shear, showing the near vanishing of the torque in the ion direction.

the foot of the pedestal where the shear is significantly reduced by the presence of a strong bootstrap current driven by the pressure gradient in the pedestal. In the presence of such a weak shear region, the reconnection time is increased, so that the effect of the diamagnetic drifts is more pronounced. Our calculations show that this leads to a pronounced asymmetry of the force curve, creating a band of frequencies in the ion direction where the force is negligible (Fig. 5). If the force actually vanished for a finite value of the frequency, this flux surface would be immune to locked-mode onset, since the condition for the balance between the viscous and resonant electromagnetic force could then always be satisfied. Given the numerous other rational surfaces in the edge, it is doubtful whether this possibility is of any importance in explaining the RMP observations, but it may be of interest in other circumstances, such as for low-density locked modes in reversed shear discharges. 
Role of Singular Layers in the Plasma Response to Resonant Magnetic Perturbations19

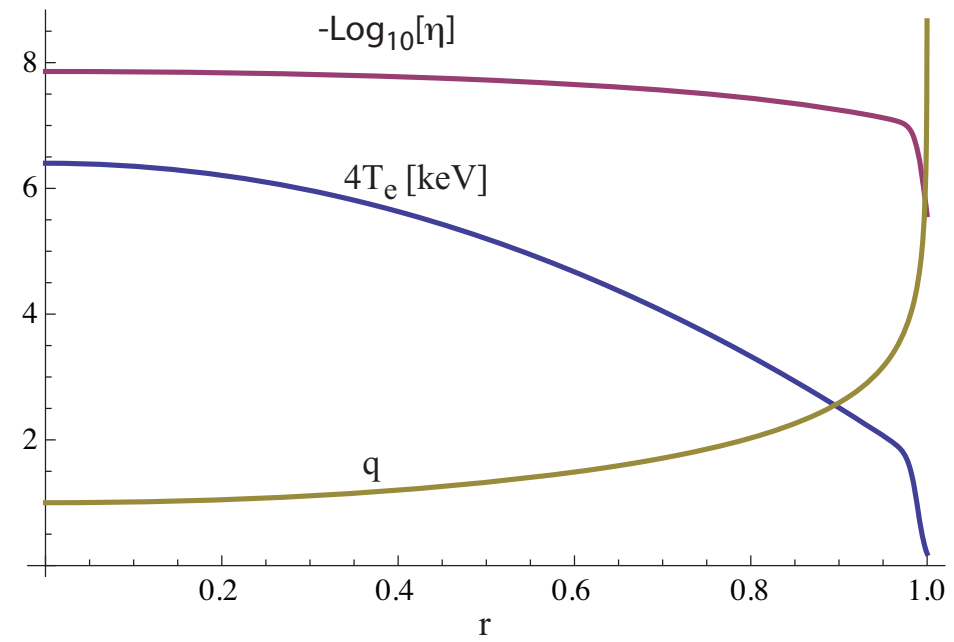

Figure 6. (color online) Profiles of the logarithm of the resistivity $\eta$, the electron temperature $T_{e}$, and the safety factor $q$ as a function of the normalized minor radius $r$.

\section{Application to a model for an H-mode pedestal}

In this section, we examine the predictions of the theory described above when applied to a model of an H-mode pedestal that is representative of the DIII-D experiment [62]. The model used here is similar to but distinct from that of Ref. [62]. We consider the following parameters: a minor radius $a=0.6 \mathrm{~m}$, a major radius $R_{0}=1.7 \mathrm{~m}$, a toroidal field on axis $B_{0}=1.95 \mathrm{~T}$, and a flat density profile with $n_{e}=3.5 \cdot 10^{19} \mathrm{~m}^{-3}$. We model the temperature by

$$
T_{e}(r)=T_{e 0}\left\{1-\tanh \left[\left(r-r_{0 T}\right) / \delta r_{0 T}\right]\right\}\left(1-k_{2} r^{2}\right) / 2,
$$

where all the radial variables are normalized to $a$ and $T_{e 0}=1.6 \mathrm{keV} ; r_{0 T}=0.99 ; \delta r_{0 T}=$ $0.01 ;$ and $k_{2}=.75$.

For the safety factor profile, we use the following combination of log functions

$$
q(r)=1 .-1.2 \log \left(1-r^{2}\right)+0.3 r^{2} \log \left(1-r^{2}\right) .
$$

This profile takes into account the effect of the separatrix on the magnetic shear, an effect that affects the plasma response in the singular layer but cannot be properly modeled with an initial-value cylindrical code because the corresponding current profile is unstable to tearing modes. The electron temperature and safety-factor profiles are shown in Fig. 6. Lastly, for the profile of the radial electric field we use the following model:

$$
E_{r}(r)=\hat{E}_{r}(r) \frac{\left(1-k_{4} r^{2}\right)^{2}}{\left(1-k_{4}\right)^{2}}
$$

where $k_{4}=0.75$ and $\hat{E}(r)$ describes the variation of the electric field in the edge through a linear combination of a tanh function and a Gaussian:

$$
\hat{E}_{r}(r)=\left\{E_{1}\left(1+\tanh \left[-\frac{r-r_{1 E}}{\delta r_{1 E}}\right]\right)+E_{2} \exp \left[-\left(\frac{r-r_{2 E}}{\delta r_{2 E}}\right)^{2}\right]\right\},
$$


Role of Singular Layers in the Plasma Response to Resonant Magnetic Perturbations20

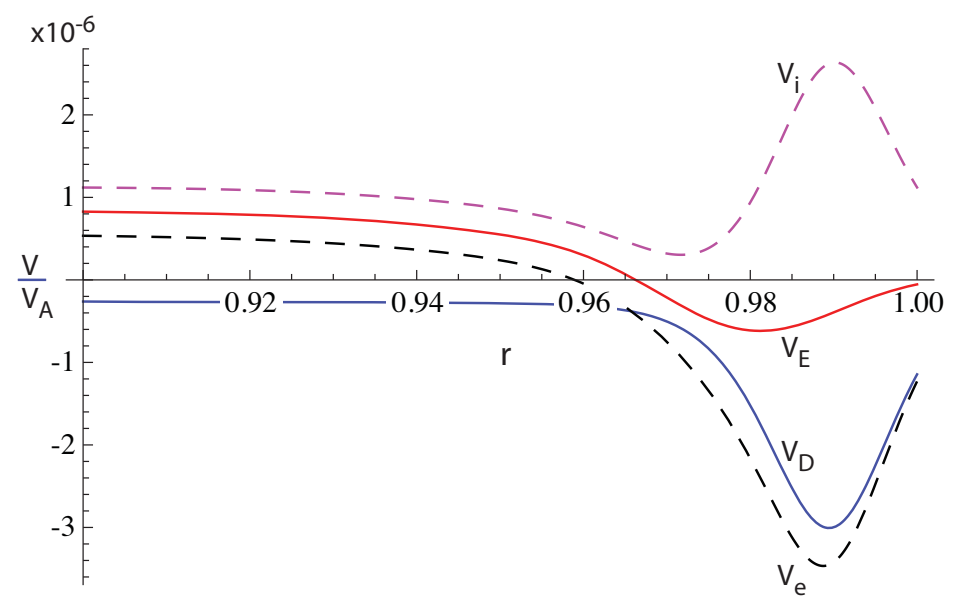

Figure 7. Profiles of the $\mathbf{E} \times \mathbf{B}$, diamagnetic $\left(V_{D}\right)$, and perpendicular electron $\left(V_{e}\right)$ and ion $\left(V_{i}\right)$ velocities in the edge.

where $r_{1 E}=0.96, \delta r_{1 E}=0.03, r_{2 E}=0.98 \delta r_{2 E}=0.015, E_{1}=4.0 \mathrm{~V} / \mathrm{m}$, and $E_{2}=-10.0$ $\mathrm{V} / \mathrm{m}$. The corresponding profiles for the perpendicular velocity of the electrons $\left(V_{e}\right)$, guiding centers $\left(V_{E}\right)$, and ions $\left(V_{i}\right)$ in the edge are shown in Fig. 7, and the global profile of the $n=1$ Doppler frequency is shown in Fig. 10. Note that with these profiles, the ion-sound Larmor radius $\rho_{s}$ is approximately twice the width of the tearing layer, so that two-fluid effects such as parallel electron compressibility are important.

Fig. 8 shows the reconnection quotient $S_{m}=B_{n} / B_{n}^{\text {full }}=A / A^{\text {full }}=|\hat{\Delta}|^{-1}$ evaluated from the layer theory. Here $B_{n}$ is the perturbed magnetic field normal to the equilibrium flux surface, and $A^{\text {full }}$ is the amount of flux that would reconnect in the absence of plasma rotation. In general, $A^{\text {full }}$ is larger but comparable to $A^{\text {vac }}$, the vacuum perturbation, due to the amplifying effect of gradients in the equilibrium pressure and current. The reconnection quotient is very small except at the electron reversal surface and at the foot of the pedestal. It is particularly small in the steepest part of the pedestal, due to the increased suppression caused by the strong diamagnetic flows. In the center of the pedestal the suppression factor is of order $10^{-4}$, corresponding to islands that are only a few percent of their vacuum widths.

Fig. 9 shows the quasilinear electromagnetic force acting on the plasma in the resonant layers normalized to the squared amplitude of the screening current. The force diverges and changes sign at the electron reversal surface, as expected. It also becomes large at the foot of the pedestal due to the reduced perpendicular velocity and the increased collision frequency. The combination of the strong braking force, the substantial reconnection quotient and the vanishing separation between successive resonant surfaces leads to the expectation that the foot of the pedestal will exhibit magnetic chaos.

In order to calculate the change in the velocity profile, we sum the products of the resonant forces with the changes in velocity caused by unit forces located at the resonances. These are obtained by solving the momentum transport equation with 


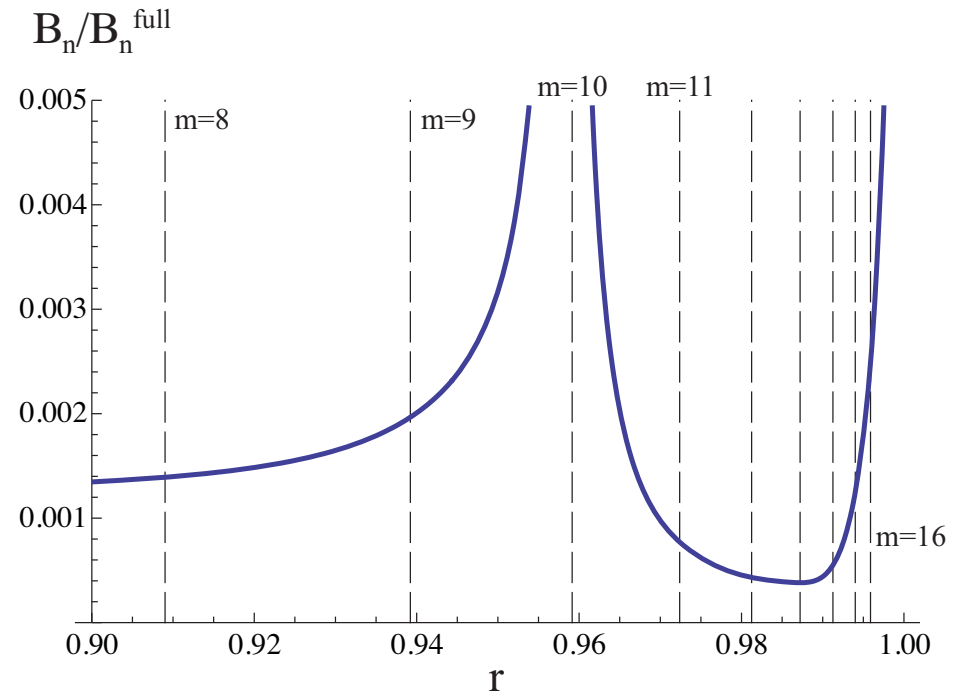

Figure 8. (color online) Reconnection quotient $B_{n} / B_{n}^{\text {full }}=A / A^{\text {full }}$ for the chosen profiles. The vertical lines represent the mode-rational surfaces for $n=3$. Presently available theory leads to the expectation that the plasma rotation at the $q=10 / 3$ resonant surface near the electron velocity-reversal surface should lock to the RMP field, eliminating the suppression of reconnection by plasma flows and allowing the island to grow to its full amplitude.

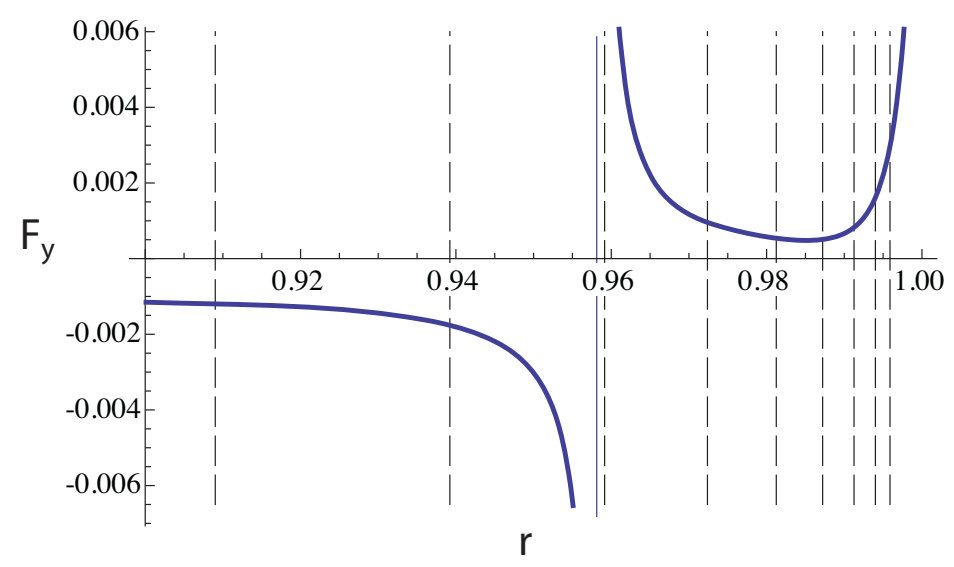

Figure 9. Normalized force $F_{y}$ acting on the plasma at the resonant surfaces, as a function of the minor radius $r$. Note the divergence at the electron velocity reversal surface and the comparatively small value of the force in the pedestal. The resonant forces brake the plasma rotation to the left of the reversal surface, but accelerate it to the right, in the pedestal region. 
a delta-function force applied at the resonant surface of interest and with a constant viscosity coefficient. In cylindrical geometry the corresponding change in velocity $\delta \hat{V}_{m}(r)$ takes the form

$$
\delta \hat{V}_{m}(r)=-\frac{r_{m}}{\mu} \begin{cases}\log \left(r_{m}\right), & r<r_{m} \\ \log (r), & r>r_{m}\end{cases}
$$

where the resonant radii satisfy $n q\left(r_{m}\right)=m$. The total velocity change, taking into account poloidal rotation damping, is then

$$
\delta \hat{V}=\frac{2 n}{R_{0} \mu_{0} m_{i} n_{e 0} a} \sum_{m} r_{m}^{2}\left|A^{\text {full }}\right|^{2} \Im\left[1 / \Delta\left(r_{m}\right)\right] \delta \hat{V}_{m}(r),
$$

where $A^{\text {full }}$ should in principle be obtained by solving the mode equation for the chosen current and pressure profiles. For simplicity, we use instead the vacuum amplitudes of the various harmonics as calculated by the SURFMN code for the I-coils on DIII-D.

The singularity of the braking force at the reversal surface presents a difficulty, however, when calculating the change in velocity caused by the RMP, since the resonant surface closest to the electron velocity reversal surface dominates the response. In fact, the quasilinear evaluation of the change in velocity is inapplicable at the closest resonant surface, since the force at that surface is well in excess of that needed to lock the plasma rotation. We thus assume that the rotation is locked at that surface, and evaluate the force applied by the RMP to maintain the locked mode by requiring that the electron velocity vanish at the $q=10 / 3$ surface, consistent with the trapping of the electrons in the locked island. That is, we replace $F_{y}\left(r_{10}\right)$ in Eq. 43 by a value evaluated so that $v_{e}\left(r_{10}\right)=0$. At all other surfaces we use quasilinear theory to evaluate the resonant forces. Fig. 10 shows the resulting change in the Doppler frequency $\delta \omega_{E}=\mathbf{k} \cdot \delta \mathbf{V}_{E}$, as well as the final velocity $\omega_{E}^{\mathrm{RMP}}$. The change in velocity is qualitatively consistent with observations. In particular, the plasma accelerates in the edge and brakes in the core, corresponding to the opposite sign of the electron rotation in these two regions.

\section{Summary}

We have examined the response of the plasma edge to Resonant Magnetic Perturbations by applying a two-fluid model to the singular layers centered at the resonant surfaces. We have used this model to calculate the island reconnection quotients, the plasma acceleration, and the density pumpout for parameters representative of an H-mode edge plasma. Unlike studies using cylindrical models, in which the logarithmic singularity of the magnetic shear that characterizes the separatrix must be modeled by the use of artificially peaked current profiles, the asymptotic, singular-layer approach used in the present paper makes it possible to evaluate the plasma response for any profile of the safety factor. Furthermore, unlike 3D nonlinear initial-value codes, the singularlayer analysis makes it possible to use values of the resistivity corresponding to the experimentally measured plasma parameters. This is highly desirable from the point 
Role of Singular Layers in the Plasma Response to Resonant Magnetic Perturbations23

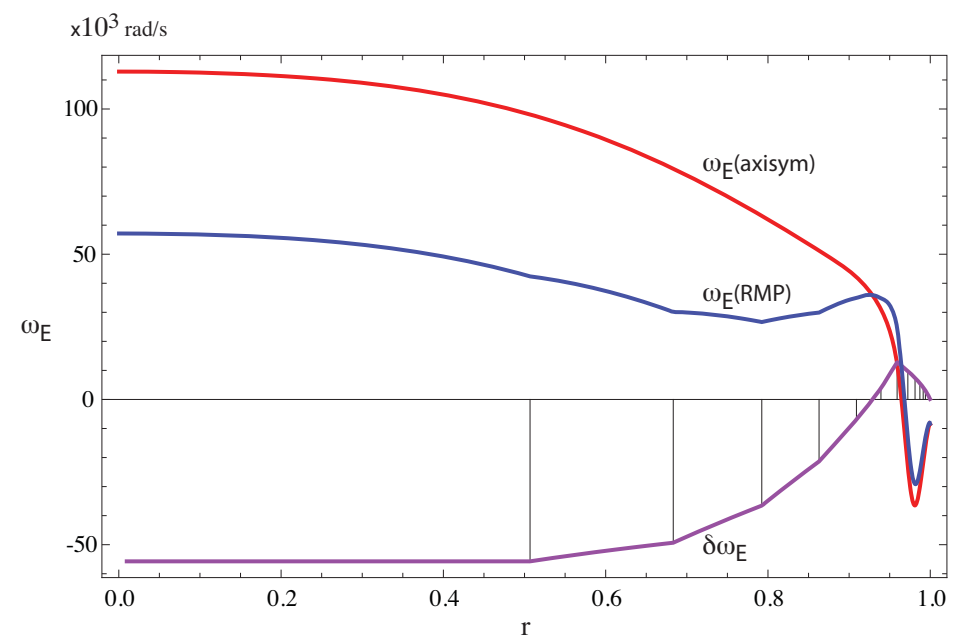

Figure 10. Velocity profiles before (axisym) and after (RMP) the application of the RMP, and the difference between these velocity profiles calculated from the resonant forces at the rational surfaces.

of view of interpreting the experimental results, since low collisionality is known to be necessary for the complete suppression of ELM activity [13, 14].

The degree to which the rotation of the plasma inhibits the magnetic reconnection that is necessary to form islands, such as those that appear in Poincaré maps and are the root cause of magnetic chaos, is described by the reconnection quotients $S_{m}$, where $m=n q$ labels the resonant surfaces. The screening factor is defined as the ratio of the amplitude of the perturbed flux in a suppressed island to the perturbed flux for a fully reconnected island, such as that which theory predicts will be created when the Doppler frequency of the electron fluid, $\mathbf{k} \cdot \mathbf{v}_{e}$, matches the RMP frequency (which vanishes in most experiments) [54]. The suppression factor is given by the modulus of the inverse of the normalized matching parameter, $S_{m}=|\hat{\Delta}|^{-1}$.

Our results show that anomalous transport (viscosity and particle diffusivity) significantly decrease $S_{m}$, corresponding to a more complete suppression in the semicollisional regime, which is the relevant regime in the edge of experiments with small collisionality, such as the ITER-similar DIII-D discharges examined here. On the other hand, the parallel electron compressibility, which is omitted from the MHD model, acts to screen the parallel electric fields and to concentrate the current in a layer of width less than the Larmor radius [80]. Neglecting parallel compressibility weakens the suppression, and can compensate to some degree the neglect of the effects of anomalous transport.

The resonant forces that the RMP exerts on the plasma are proportional to the imaginary part of the inverse of the matching parameter, $\Im\left(\left.\hat{\Delta}\right|^{-1}\right)$. Since the phase of $\hat{\Delta}$ is generally close to or equal to $\pi / 2$, the normalized electromagnetic force acting on the plasma at the resonant surfaces is generally comparable in magnitude to the island suppression factor. As a result, the resonant torque and the density pumpout can both be expected to be small when the suppression is strong $\left(S_{m} \ll 1\right)$. This is the case, in 
Role of Singular Layers in the Plasma Response to Resonant Magnetic Perturbations24

particular, in the center of the pedestal.

An important feature of the experiments is the presence of a surface where the perpendicular component of the electron velocity reverses. Theory predicts that a locked mode should form easily when this surface crosses a mode-rational surface $(q=m / n)$ in the course of the normal evolution of the profile during a shot. The absence of the signatures of locked-mode onset, such as abrupt rotation changes and magnetic impulses visible on magnetic sensors outside the plasma, could be due to the fact that the natural rotation velocity of the plasma is already small. An alternative explanation is suggested by the observations of the locking threshold in TEXTOR, which show that a substantial threshold persists even when the electron perpendicular velocity vanishes [55, 56]. A theoretical explanation for these observations remains to be proposed.

Acknowledgment The authors are grateful to P. Beyer, K. Burrell, T. Evans, and Q. $\mathrm{Yu}$ for informative discussions. This work was funded by the US DoE contract number DE-FG03-96ER-54346 and US DoE/LLNL Contract DE-AC52-07NA27344

\section{Appendix A: Collisional regimes: $\rho \ll 1$}

For sufficiently high collisionality, the layer width exceeds the ion-sound Larmor radius $\rho_{s}$ and the layer equation reduces to

$$
\frac{d}{d k}\left(\frac{k^{2}}{k^{2}+Q_{e}} \frac{d \hat{\Upsilon}}{d k}\right)-\left(\frac{Q Q_{i}+Q_{i}(D+P) k^{2}+P D k^{4}}{Q_{e}+D k^{2}}\right) k^{2} \hat{\Upsilon}=0 .
$$

Analytic solutions of this equation are known for particular regimes. We briefly review these here.

\section{Ideal regime:}

In the ideal regime the response is localized to the region $k^{2} \ll Q_{e}$. Substituting $\hat{\Upsilon}=\hat{\chi} / k$, the layer equation becomes

$$
\frac{d^{2} \hat{\chi}}{d k^{2}}-\left(\frac{Q Q_{i}+Q_{i}(D+P) k^{2}+P D k^{4}}{1+D k^{2} / Q_{e}}\right) \hat{\chi}=0 .
$$

For $\mathrm{D}=0$ this equation can be solved as follows. Changing variables according to $u=\sqrt{Q_{i} P} k^{2}$ and $\hat{\chi}=e^{-u / 2} \hat{w}$, the equation becomes

$$
u \hat{w}^{\prime \prime}+\left((1 / 2-u) \hat{w}^{\prime}-\frac{1}{4}\left[1-\left(\frac{Q^{2} Q_{i}}{P}\right)^{1 / 2}\right] \hat{w}=0 .\right.
$$

The solution that is well-behaved at large $k$ is

$$
\hat{\chi}(u)=e^{-u / 2} U(a / 4,1 / 2, u),
$$


Role of Singular Layers in the Plasma Response to Resonant Magnetic Perturbations25

where $U$ is the confluent Hypergeometric function of the second kind and $a=1-$ $\left[Q^{2} Q_{i} / P\right]^{1 / 2}$. Using the series expansion of $U$ at small $k$, we find that

$$
\hat{\Delta}(k)=-\frac{\pi}{2} \frac{\Gamma\left(\frac{1}{4}-\frac{1}{4}\left(\frac{Q^{2} Q_{i}}{P}\right)^{1 / 2}\right)}{\Gamma\left(\frac{3}{4}-\frac{1}{4}\left(\frac{Q^{2} Q_{i}}{P}\right)^{1 / 2}\right)}\left(Q_{i} P\right)^{-1 / 4}
$$

For $Q^{2} Q_{i} \ll P$, the dispersion relation reduces to

$$
\hat{\Delta}(k)=-\frac{\pi}{2} \frac{\Gamma\left(\frac{1}{4}\right)}{\Gamma\left(\frac{3}{4}\right)}\left(Q_{i} P\right)^{-1 / 4}
$$

This is the inviscid resistive regime. In this regime the mode width is of order $\delta k \simeq\left(Q_{i} P\right)^{-1 / 4}$.

In the opposite limit, $Q^{2} Q_{i} \gg P$, the dispersion relation is

$$
\hat{\Delta}(k)=-\frac{\pi}{\sqrt{Q Q_{i}}}
$$

This is the ideal inertial regime. The mode width is $\delta k \simeq\left(Q Q_{i}\right)^{-1 / 2}$.

\section{Inviscid regime}

The inviscid regime corresponds to $P=D=0$.

$$
\frac{Q_{e}}{Q Q_{i}} \frac{d}{d k}\left(\frac{1}{k^{2}} \frac{d \hat{J}}{d k}\right)-\frac{k^{2}+Q_{e}}{k^{2}} \hat{J}=0 .
$$

This equation has the solution

$$
\hat{J}=e^{-u / 2} U\left(-\frac{1}{4}\left(1-\sqrt{Q Q_{i} Q_{e}}\right),-\frac{1}{2}, u\right),
$$

where $u=\left(Q Q_{i} / Q_{e}\right)^{1 / 2} k^{2}$. The matching procedure yields

$$
\hat{\Delta}=-\frac{\pi}{8} \frac{\Gamma\left(\frac{1}{4}\left(\left(Q Q_{i} Q_{e}\right)^{1 / 2}-1\right)\right)}{\Gamma\left(\frac{1}{4}\left(\left(Q Q_{i} Q_{e}\right)^{1 / 2}+5\right)\right)}\left(Q Q_{i}\right)^{1 / 4} Q_{e}^{3 / 4} .
$$

For $Q Q_{i} Q_{e} \gg 1$, we recover the ideal inertial result already obtained in the previous subsection. The opposite limit, $Q Q_{i} Q_{e} \ll 1$, describes the inviscid resistive regime,

$$
\hat{\Delta}=\frac{\pi}{2} \frac{\Gamma\left(\frac{3}{4}\right)}{\Gamma\left(\frac{5}{4}\right)}\left(Q Q_{i}\right)^{1 / 4} Q_{e}^{3 / 4} .
$$

In this regime the mode width is $\delta k=\left(Q_{e} / Q Q_{i}\right)^{1 / 4}$.

\section{Visco-resistive regime}

We conclude the analysis of the collisional regimes by considering the case when both resistivity and viscosity are important. In this case the layer divides into two regions. For small $k$, the equation reduces to

$$
\frac{d}{d k}\left(\frac{k^{2}}{k^{2}+Q_{e}} \frac{d \hat{\Upsilon}}{d k}\right)=0
$$


Role of Singular Layers in the Plasma Response to Resonant Magnetic Perturbations26

with the solution

$$
\Upsilon=-\frac{Q_{e}}{k}+k+\alpha,
$$

where $\alpha$ is an unknown integration constant to be determined by matching to the solution at large $k$. The equation for large $k$ takes the form

$$
\frac{d^{2} \hat{\Upsilon}}{d k^{2}}-\frac{(P+D) Q_{i}}{Q_{e}} k^{4} \hat{\Upsilon}=0 .
$$

The solution is

$$
\Upsilon=e^{-u / 2} U\left(\frac{1}{3}, \frac{2}{3}, u\right),
$$

where $u=2\left((P+D) Q_{i} / Q_{e}\right)^{1 / 2} k^{3} / 3$. The matching parameter is

$$
\hat{\Delta}=\pi 6^{2 / 3} \frac{\Gamma\left(\frac{5}{6}\right)}{\Gamma\left(\frac{1}{6}\right)}\left(P Q_{i}\right)^{1 / 6} Q_{e}^{5 / 6} .
$$

\section{Appendix B: Semi-collisional regimes}

\section{Ideal semi-collisional regime}

In the ideal semi-collisional regime, $P=D=0$, the layer divides into an inner current channel and a broader layer where the current is much weaker. The mode equation may be solved separately in each region.

In the inner layer, the mode equation simplifies to

$$
\frac{d}{d k}\left[\left(\frac{Q_{e}+Q_{i} \rho^{2} k^{2}}{Q Q_{i} k^{2}}\right) \frac{d \hat{J}}{d k}\right]=0 .
$$

The solution is

$$
\hat{J}=C_{1} \frac{Q}{\rho^{3}} \sqrt{\frac{Q_{e}}{Q_{i}}}[u-\arctan (u)]+C_{2},
$$

where $u=k \rho \sqrt{Q_{i} / Q_{e}}$.

In the outer layer, the equation reduces to

$$
\frac{\rho^{2}}{Q} \frac{d^{2} \hat{J}}{d k^{2}}-\hat{J}=0 .
$$

with solution

$$
\hat{J}=\exp (-|k| \sqrt{Q} / \rho) .
$$

Matching these two solutions determines the ratio of the integration constants $C_{j}$ :

$$
\frac{C_{2}}{C_{1}}=\frac{\pi}{2} \sqrt{\frac{Q_{e}}{Q_{i}}} \frac{Q}{\rho^{3}}-\frac{\sqrt{Q}}{\rho} .
$$


Lastly, matching the small- $k$ asymptotic expansion in Eq. A.11 to Eq. 26) and using the definition of $\hat{\Delta}$, Eq. 14 , yields the desired result:

$$
\hat{\Delta}=\pi \frac{Q_{e} \sqrt{Q}}{\rho}\left(1-\frac{\pi}{2 \rho^{2}} \sqrt{\frac{Q Q_{e}}{Q_{i}}}\right)
$$

The first term in Eq. A.14 represents the standard semi-collisional result, and the second term is a correction due to polarization currents flowing outside of the central electron channel. This correction can be important when the constant- $A$ approximation is violated, as may occur at large $\beta$, but we have found that it is generally small for the range of experimental parameters we have examined.

\section{Diffusive semi-collisional regime}

The presence of diffusion modifies the behavior of the solution at large ballooning angle $k$, where the layer equation for the electron stream-function becomes

$$
\frac{d^{2} \hat{\Upsilon}}{d k^{2}}-\frac{D k^{2}}{(1+\tau) \rho^{2}} \hat{\Upsilon}=0
$$

Changing variables to $u=\left[D / \rho^{2}(1+\tau)\right]^{1 / 2} k^{2}$ and $\hat{\Upsilon}=w(u) \exp (-u / 2)$, the equation takes the form of the confluent Hypergeometric equation. The solution with the appropriate decaying behavior for $k \rightarrow \infty$ is

$$
w(u)=U(1 / 4,1 / 2, u) .
$$

Expanding this solution for small $k$, we find

$$
\hat{\Upsilon}=\pi\left[\frac{1}{\Gamma(3 / 4) \Gamma(1 / 2)}-u^{1 / 2} \frac{1}{\Gamma(1 / 4) \Gamma(3 / 2)}\right] .
$$

Matching to the small- $k$ asymptotic solution yields the dispersion relation,

$$
\hat{\Delta}(Q)=2 \pi \frac{\Gamma\left(\frac{3}{4}\right)}{\Gamma\left(\frac{1}{4}\right)}\left(\frac{D}{\rho^{2}(1+\tau)}\right)^{1 / 4} Q_{e} .
$$

This dispersion relation agrees well with the numerical solutions in its regime of validity. 
Role of Singular Layers in the Plasma Response to Resonant Magnetic Perturbations28

\section{References}

[1] HAWRYLUK, R. et al., Nuclear Fusion 49 (2009) 065012 (15pp).

[2] EVANS, T. E. et al., Nat. Phys. 2 (2006) 419.

[3] WAELBROECK, F. L., Nuclear Fusion 49 (2009) 104025 (15pp).

[4] SUTTROP, W. et al., Nuclear Fusion 45 (2005) 721.

[5] BURRELL, K. H. et al., Physics of Plasmas 12 (2005) 056121.

[6] SNYDER, P. et al., Nuclear Fusion 47 (2007) 961.

[7] COLTON, A. L. et al., Nucl. Fusion 39 (1999) 551.

[8] SHOJI, T. et al., Journal of Nuclear Materials 196-198 (1992) 296 .

[9] CANIK, J. M. et al., Phys. Rev. Lett. 104 (2010) 045001.

[10] LIANG, Y. et al., Physical Review Letters 98 (2007) 265004.

[11] LIANG, Y. et al., Plasma Physics and Controlled Fusion 49 (2007) B581.

[12] EVANS, T. E. et al., Phys. Rev. Lett. 92 (2004) 235003.

[13] BURRELL, K. H. et al., Plasma Physics and Controlled Fusion 47 (2005) B37.

[14] EVANS, T. E. et al., Physics of Plasmas 13 (2006) 056121.

[15] EVANS, T. et al., Nuclear Fusion 48 (2008) 024002 (10pp).

[16] SCHAFFER, M. et al., Nuclear Fusion 48 (2008) 024004 (14pp).

[17] BECOULET, M. et al., Nuclear Fusion 48 (2008) 024003 (16pp).

[18] TOKAR, M. Z. et al., Physical Review Letters 98 (2007) 095001.

[19] FENSTERMACHER, M. E. et al., Phys. Plasmas 15 (2008) 056122.

[20] TOKAR, M. et al., Nuclear Fusion 48 (2008) 024006 (9pp).

[21] FENSTERMACHER, M. et al., Nuclear Fusion 48 (2008) 122001 (4pp).

[22] NARDON, E. et al., J. Nucl. Mater. 363-365 (2007) 1071.

[23] SCHMITZ, O. et al., Physical Review Letters 103 (2009) 165005.

[24] JOSEPH, I. et al., Journal of Nuclear Materials 363-365 (2007) 591.

[25] JOSEPH, I. et al., Nucl. Fusion 48 (2008) 045009.

[26] FRERICHS, H. et al., Nuclear Fusion 50 (2010) 034004.

[27] TOKAR, M. Z. et al., Physics of Plasmas 15 (2008) 072515.

[28] HEYN, M. F. et al., Nuclear Fusion 46 (2006) S159.

[29] PARK, G. et al., Physics of Plasmas 17 (2010) 102503.

[30] IZZO, V. et al., Nucl. Fusion 48 (2008) 115004 (9pp).

[31] STRAUSS, H. et al., Nuclear Fusion 49 (2009) 055025 (8pp).

[32] REISER, D. et al., Physics of Plasmas 16 (2009) 042317.

[33] HENDER, T. C. et al., Nucl. Fusion 32 (1992) 2091.

[34] LA HAYE, R. J. et al., Nucl. Fusion 32 (1992) 2119.

[35] LA HAYE, R. J. et al., Phys. Fluids B 4 (1992) 2098.

[36] MORRIS, A. W. et al., Phys. Fluids B 4 (1992) 413.

[37] BUTTERY, R. et al., Nuclear Fusion 39 (1999) 1827.

[38] BUTTERY, R. et al., Nuclear Fusion 40 (2000) 807.

[39] LA HAYE, R. J. et al., Nucl. Fusion 33 (1993) 349.

[40] LA HAYE, R. J. et al., Phys. Plasmas 1 (1994) 373.

[41] FITZPATRICK, R., Physics of Plasmas 5 (1998) 3325.

[42] FISHPOOL, G. M. et al., Nucl. Fusion 34 (1994) 109.

[43] FITZPATRICK, R. et al., Phys. Fluids B 3 (1991) 644.

[44] PARKER, R. D., Proceedings of the 19th EPS-ICPP conf., Innsbruck, 1992, edited by K. Bethge

(European Physical Society, Geneva, 1993), 1 (1992) 427.

[45] FITZPATRICK, R., Nuclear Fusion 33 (1993) 1049.

[46] FITZPATRICK, R. et al., Phys. Plasmas 1 (1994) 3337.

[47] BOOZER, A., Phys. Plasmas 3 (1996) 4620.

[48] KIKUCHI, Y. et al., Plasma Physics and Controlled Fusion 48 (2006) 169. 
[49] SHAING, K. C. et al., Physics of Fluids 26 (1983) 3315.

[50] LAZZARO, E. et al., Phys. Plasmas 9 (2002) 3906.

[51] ZHU, W. et al., Phys. Rev. Lett. 96 (2006) 225002.

[52] COLE, A. J. et al., Physical Review Letters 99 (2007) 065001.

[53] BECOULET, M. et al., Nuclear Fusion 49 (2009) 085011 (13pp).

[54] WAELBROECK, F. L., Phys. of Plasmas 10 (2003) 4040.

[55] KOSLOWSKI, H. R. et al., Plasma Phys. Control. Fusion 48 (2006) B53B61.

[56] KOSLOWSKI, H. et al., Nucl. Fusion 46 (2006) L1.

[57] COLE, A. et al., Physics of Plasmas 13 (2006) 032503.

[58] WOLFE, S. M. et al., Physics of Plasmas 12 (2005) 056110.

[59] KIKUCHI, Y. et al., Plasma Physics and Controlled Fusion 49 (2007) A135.

[60] YU, Q. et al., Nucl. Fusion 48 (2008) 024007.

[61] HEYN, M. F. et al., Nucl. Fusion 48 (2008) 024005.

[62] NARDON, E. et al., Nuclear Fusion 50 (2010) 034002.

[63] YU, Q. et al., Physics of Plasmas 16 (2009) 042301.

[64] YU, Q. et al., Nuclear Fusion 49 (2009) 062001 (5pp).

[65] MiLitello, F. et al., Nuclear Fusion 49 (2009) 065018 (11pp).

[66] NARDON, E. et al., Phys. Plasmas 14 (2007) 092501.

[67] LIU, Y. et al., Physics of Plasmas 17 (2010) 122502.

[68] HAZELTine, R. D. et al., Phys. Reports 121 (1985) 1.

[69] TASSI, E. et al., Plasma Physics and Controlled Fusion 50 (2008) 085014 (29pp).

[70] GRASSO, D. et al., Phys. of Plasmas 17 (2010) 082312.

[71] TASSI, E. et al., J. Phys.: Conf. Series 260 (2010) 012020.

[72] CHANG, C. S. et al., Self-consistent simulation of kinetic pedestal transport under RMP penetration, in Fusion Energy 2010 (Proc. 23 Int. Conf. Daejon 2010) CD-ROM file THC/P404, IAEA, Vienna, 2010.

[73] CONNOR, J. W. et al., Physics of Fluids 30 (1987) 3180.

[74] PEgORARO, F. et al., Plasma Physics and Controlled Fusion 28 (1986) 647.

[75] BEYER, P. et al., Plasma Physics and Controlled Fusion 53 (2011) 054003.

[76] IVANOV, I. B. et al., JETP Letters 86 (2007) 364.

[77] WAELBROECK, F. L., Plasma Phys. Control. Fusion 49 (2007) 905.

[78] GROEBNER, R. et al., Nuclear Fusion 44 (2004) 204.

[79] SNYDER, P. B. et al., Physics of Plasmas 16 (2009) 056118.

[80] DRAKE, J. F. et al., Phys Fluids 26 (1983) 2509. 